$\operatorname{IPPP} / 04 / 13$

DCPT $/ 04 / 26$

15th March 2004

\title{
Double-diffractive $\chi$ meson production at the hadron colliders
}

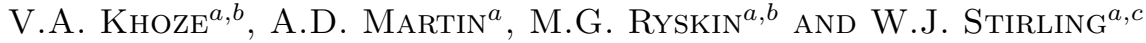 \\ ${ }^{a}$ Department of Physics and Institute for Particle Physics Phenomenology, \\ University of Durham, DH1 3LE, UK \\ ${ }^{b}$ Petersburg Nuclear Physics Institute, Gatchina, St. Petersburg, 188300, Russia \\ ${ }^{c}$ Department of Mathematical Sciences, University of Durham, DH1 3LE, UK
}

\begin{abstract}
The double-diffractive production of $\chi_{c}$ and $\chi_{b}$ mesons, with a rapidity gap on either side, is studied, using both the Regge formalism and the perturbative QCD approach. Due to the rather low scale, the exclusive double-diffractive process $p p \rightarrow p+\chi+p$ is predicted to dominate, whereas the probability that the incoming protons dissociate is expected to be relatively small. We evaluate the corresponding $\chi$ production cross sections at the Tevatron and LHC energies. For the double-diffractive process with proton dissociation, it is possible to select events with large transverse momenta transferred through the rapidity gaps, by measuring the transverse energy, $E_{\perp}$, flows in the proton fragmentation regions. Then the large $E_{\perp}$ provides a scale to justify the use of perturbative QCD, and to allow a spin-parity analysis of the centrally produced system to be performed, by studying the azimuthal angular correlations between the directions of the forward and backward $E_{\perp}$ flows. The central production of the new $X(3872)$ charmonium state is considered.
\end{abstract}

\section{Introduction}

Central exclusive double-diffractive processes have traditionally been considered as a promising way to study new (and old) particles in an especially clean environment, see, for example, Ref. [1]. In addition they give information about the structure of the Pomeron and of the mechanism of Pomeron-Pomeron fusion. Here we are particularly interested in the double-diffractive production of, C-even, heavy quarkonium $\left(\chi_{c}, \chi_{b}\right)$ at the Tevatron and the LHC [2] 3, see also Refs. 4] 5. Since the early days of QCD, heavy quark production has been a fertile testing ground of many aspects of the theory. More recently, there has been much activity in studying the long standing discrepancy between the NLO predictions and the data for heavy quark production at hadron colliders [6]. Heavy quarkonium production is a valuable tool, since it provides important information on the physics of bound states and, in particular, allows a test of the ideas and methods of QCD effective field theories, which is one of the most popular recent approaches; for reviews and references see, for example, 7 . 
(a)

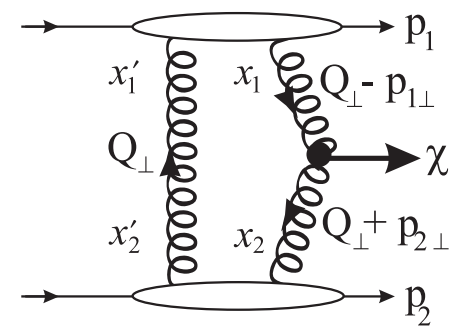

(b)

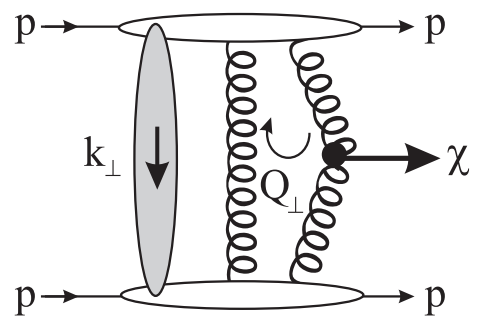

Figure 1: (a) The QCD diagram for double-diffractive exclusive production of a $\chi\left(0^{+}\right)$meson, $p p \rightarrow p+\chi+p$, where the gluons of the hard subprocess $g g \rightarrow \chi$ are colour screened by the second $t$-channel gluon. (b) The rescattering or absorptive corrections to $p p \rightarrow p+\chi+p$, where the shaded region represents the soft $p p$ rescattering corrections, leading to the suppression factor $\hat{S}^{2}$.

Detailed experimental and theoretical studies of double-diffractive $\chi$ production offer a new window on this topic, and may shed light on some of the unresolved issues. These processes have some surprising features. In order to gain insight into the problems encountered in the evaluation of these processes, we first recall an analogous process, which in many respects is theoretically simpler. Namely, the exclusive double-diffractive central production of Higgs bosons, which has been advocated as a good way to study the Higgs sector at the LHC $[\underline{8}$. Both the $\chi$ and Higgs production processes may be written in the form

$$
p p \rightarrow p+(\chi \text { or } H)+p,
$$

where the + signs are used to represent the presence of large rapidity gaps. Since the Higgs is expected to have mass, $M_{H}$, about $120 \mathrm{GeV}$ or more, its production cross section may be calculated perturbatively, via the diagram shown in Fig. 1. The hard subprocess $g g \rightarrow H$ is initiated by gluon-gluon fusion, and the second $t$-channel gluon is needed to screen the colour. The crucial observation is that the integration over $Q_{\perp}$, going round the gluon loop, is made infrared safe by Sudakov factors which ensure that the subprocess gluons remain untouched in their evolution up to the hard scale $M_{H} / 2$. Provided forward proton taggers are installed, the process has the advantage that the Higgs mass may be measured in two independent ways: first from its decay products and second by observing the forward protons which accurately determine the 'missing mass' $M_{H}$. The predicted event rate and the signal-to-background ratio make such a Higgs search feasible at the LHC, but unfortunately the rate is estimated to be too small for this process to be of use at the Tevatron $[9,8$.

On the other hand the rate of double-diffractive $\chi$ production at the Tevatron is expected to be much larger. Indeed preliminary studies indicate that such events may have already been detected 10. Unfortunately such data cannot provide a reliable check on the perturbative prediction of double-diffractive Higgs production. Unlike the Higgs, the $\chi_{c}$, and to a lesser extent the $\chi_{b}$, are not sufficiently massive to make the doublediffractive cross section predictions infrared stable. Moreover, the predictions depend on the gluon distribution to the fourth power in the region $x \sim 10^{-4}$ and $Q^{2} \sim 1 \mathrm{GeV}^{2}$, where it is not sufficiently reliably known. Nevertheless, motivated by the forthcoming experimental data, and by special features of the production process, it is informative to study double-diffractive $\chi_{c}$ and $\chi_{b}$ production in more detail. In addition, the interest in double-diffractive $\chi_{b}$ (and even $\chi_{c}$ ) production has recently increased, since these processes may be measured at the LHC with luminosities of $10^{30}-10^{31} \mathrm{~cm}^{-2} \mathrm{sec}^{-1}$ by the CMS and TOTEM collaborations, with the special optics of the TOTEM detector [11. These forthcoming, and planned, measurements will sharpen our understanding of the physics of these types of processes, as well as helping to find better ways to select doublediffractive events.

Since $M_{\chi}$ is not large enough to justify the use of perturbative QCD, we will start our discussion using a Regge framework. We show how this formalism can be matched to the perturbative approach, and we will use perturbative QCD to evaluate the Pomeron-Pomeron $\rightarrow \chi$ fusion vertex.

Besides the pure exclusive process $p p \rightarrow p+\chi+p$, we will also study central diffractive inclusive $\chi$ production 
in which both of the incoming protons are destroyed,

$$
p p \rightarrow X+\chi+Y \text {. }
$$

Recall that for a heavy centrally produced state, such as a Higgs boson, the cross section for inclusive doublediffractive production is much larger than that for exclusive double-diffractive production [9],

$$
\sigma_{\text {incl }} \gg \sigma_{\text {excl }} \text {. }
$$

The situation is quite different for the production of the relatively light $\chi$ states. In this case we expect exclusive production to dominate. The reason is explained at the end of the next Section. Another interesting feature is that we can justify the use of perturbative QCD for inclusive double-diffractive $\chi$ production by selecting events with large momentum transferred through the exchanged Pomerons (which can be measured as the tranverse energy flows, $E_{i T}$, in the proton fragmentation regions). These $E_{T}$ 's provide the large scale which clearly allows the use of the perturbative QCD formalism.

\section{The Regge framework for double-diffractive $\chi$ production}

Exclusive double-diffractive $\chi$ production by Pomeron-Pomeron fusion is shown schematically in Fig.2(a), where $p_{1,2 \perp}$ are the transverse momenta of the outgoing protons,

$$
\frac{d \sigma}{d^{2} p_{1 \perp} d^{2} p_{2 \perp} d y}=\frac{1}{2^{8} \pi^{5}} g_{N}^{2}\left(p_{1 \perp}^{2}\right) V^{2}\left(p_{1 \perp}^{2}, p_{2 \perp}^{2}\right) g_{N}^{2}\left(p_{2 \perp}^{2}\right) x_{1}^{2\left(1-\alpha_{P}\left(t_{1}\right)\right)} x_{2}^{2\left(1-\alpha_{P}\left(t_{2}\right)\right)},
$$

where $g_{N}$ is the Pomeron-nucleon coupling and $V$ is the Pomeron-Pomeron- $\chi$ fusion vertex. The Pomeron trajectory is $\alpha_{P}(t)$, with $t_{i} \simeq-p_{i \perp}^{2}$, and

$$
x_{1,2}=\frac{M_{\chi \perp}}{\sqrt{s}} e^{ \pm y}
$$

where $y$ is the c.m. rapidity of the produced $\chi$ meson, and

$$
M_{\chi \perp}^{2}=M_{\chi}^{2}+\left(\vec{p}_{1 \perp}+\vec{p}_{2 \perp}\right)^{2} .
$$

For simplicity we have neglected the real part of the Pomeron amplitude, assuming ${ }^{1}$ that $\operatorname{Re} / \operatorname{Im} \ll 1$. The coupling $g_{N}$ is known from $p p$ data,

$$
\sigma^{\text {tot }}(p p)=g_{N}^{2}(0)\left(s / m_{N}^{2}\right)^{a_{P}(0)-1},
$$

and $\alpha_{P}(t)$ can be taken either from $J / \psi$ diffractive photoproduction data or from an analysis of $p p$ data. The vertex $V$ will be discussed later.

For inclusive double-diffractive $\chi$ production (Fig. 2(c)) we treat low- and high-mass proton dissociation differently. For low-mass dissociation, say $N \rightarrow N^{*}$, we just need to replace the coupling $g_{N}(t)$ by $g_{N \rightarrow N^{*}}(t)$. On the other hand the dissociation into a high-mass system is described in terms of the triple-Pomeron interaction with coupling $g_{3 P}$, see Fig. 2(c). The corresponding cross section is

$$
\begin{array}{r}
\frac{M_{1}^{2} M_{2}^{2} d \sigma}{d M_{1}^{2} d M_{2}^{2} d^{2} p_{1 \perp} d^{2} p_{2 \perp} d y}=\frac{1}{2^{8} \pi^{7}} g_{N}^{2}(0) g_{3 P}\left(p_{1 \perp}^{2}\right) V^{2}\left(p_{1 \perp}^{2}, p_{2 \perp}^{2}\right) g_{3 P}\left(p_{2 \perp}^{2}\right)\left(\frac{M_{1}^{2} M_{2}^{2}}{s_{0}^{2}}\right)^{\alpha_{P}(0)-1} \\
\left(\frac{x_{2} s}{M_{1}^{2}}\right)^{2\left(\alpha_{P}\left(p_{1 \perp}^{2}\right)-1\right)}\left(\frac{x_{1} s}{M_{2}^{2}}\right)^{2\left(\alpha_{P}\left(p_{2 \perp}^{2}\right)-1\right)} .
\end{array}
$$

Due to the condition

$$
x_{1} x_{2} s=M_{\chi \perp}^{2},
$$

we see that the factor

$$
\left(\frac{x_{2} s}{M_{1}^{2}}\right)^{2\left(\alpha_{P}\left(p_{1 \perp}^{2}\right)-1\right)} \propto x_{1}^{2\left(1-\alpha_{P}\left(p_{1 \perp}^{2}\right)\right)},
$$

\footnotetext{
${ }^{1}$ In other words, we neglect the signature factors in (4) and (7), assuming that $\alpha_{P}(t)$ is rather close to 1 .
} 
(a)

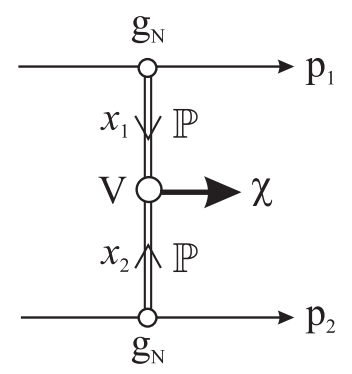

(b)

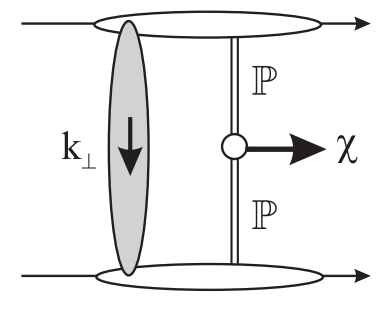

(c)

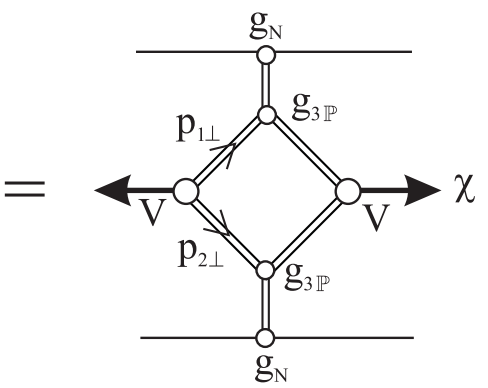

Figure 2: (a) The Pomeron-Pomeron fusion diagram for double-diffractive exclusive production of a $\chi\left(0^{+}\right)$ meson, $p p \rightarrow p+\chi+p$; see (4). (b) The rescattering or absorptive corrections to diagram (a), where the shaded region represents the soft $p p$ rescattering corrections, leading to the suppression factor $\hat{S}^{2}$. (c) The amplitude (squared) for inclusive double-diffractive $\chi$ production; the final diagram shows how high-mass diffraction is given in terms of the triple-Pomeron interaction; see (17). 
and vice-versa. Thus the cross sections of both the exclusive and inclusive diffractive dissociation processes have the same energy and rapidity dependences, see (15). For fixed masses $M_{1,2}$ (and fixed $p_{1 \perp}, p_{2 \perp}$ and $y$ ) we have

$$
d \sigma \propto s^{\alpha_{P}\left(p_{1 \perp}^{2}\right)+\alpha_{P}\left(p_{2 \perp}^{2}\right)-2} .
$$

However we have to include the absorptive corrections. The gap survival factor $\hat{S}^{2}$ - that is the probability that the rapidity gaps will not be filled by secondaries produced in the soft rescattering ${ }^{2}$ - is shown schematically by the graphs in Figs. 1(b) and 2(b). The corresponding amplitudes interfere destructively with the original bare amplitude. The elastic amplitude, shown by the shaded blob in Figs. 1(b) and 2(b), grows with energy and hence leads to a decrease of $\hat{S}^{2}$. The absorption is stronger for exclusive production (Fig. 1) since it is harder to transfer the loop momentum $k_{\perp}$ through the multiparticle chain formed by the diffractive excitations $M_{1}$ and $M_{2}[13]^{3}$.

First, we calculate the rapidity gap survival factors $\hat{S}^{2}$ for exclusive and inclusive double diffractive $\chi_{c}$ production using the formalism of Ref. [13. If we were to neglect the slope of the Pomeron trajectory, $\alpha_{P}^{\prime}=0$, then we find

$$
\begin{gathered}
\hat{S}^{2} \text { (Tevatron) }=0.05(0.15) \\
\hat{S}^{2}(\mathrm{LHC})=0.024(0.10)
\end{gathered}
$$

for exclusive (inclusive) production. When we include the Pomeron slope, $\alpha_{P}^{\prime}=0.1 \mathrm{GeV}^{2}$ (which is consistent with the HERA $J / \psi$ photoproduction data [15]), the above survival factors become

$$
\begin{gathered}
\hat{S}^{2}(\text { Tevatron })=0.07(0.16) \\
\hat{S}^{2}(\mathrm{LHC})=0.04(0.11)
\end{gathered}
$$

that is, in the Tevatron-LHC energy interval we may approximate the energy dependence by

$$
\hat{S}^{2} \propto s^{-\delta} \text { with } \delta=0.16(0.1) .
$$

For inclusive production we select events with rapidity gaps $\Delta \eta \geq 3$ : so $\alpha_{P}^{\prime}$ acts on a smaller rapidity interval, and therefore is not so effective in enlarging the value of $\hat{S}^{2}$.

Recall that the triple-Pomeron vertex $g_{3 P}(0)$ is small numerically in comparison with the elastic Pomeronnucleon coupling $g_{N}(0)$; see, for example, [16]. On the other hand $g_{3 P}$ is almost independent of the values of $p_{1 \perp}$ and $p_{2 \perp}$. Thus the suppression $\left(g_{3 P} / g_{N}\right)^{2}$ is partly compensated by the larger interval of the transverse momenta $p_{i \perp}$ sampled in diffractive dissociation ${ }^{4}$. To obtain a naive estimate of the ratio of the cross section of exclusive to inclusive $\chi$ production, we first note that the ratio of cross section for single proton dissociation to that for elastic scattering satisfies [17, 13]

$$
R=\sigma_{\mathrm{SD}} / \sigma_{\mathrm{el}}<1 / 2
$$

Thus for the 'soft' process we expect

$$
\sigma_{\text {incl }} / \sigma_{\text {excl }} \simeq R^{2}<1 / 4
$$

As a consequence, even without tagging the forward protons, we will mainly observe the exclusive $p p \rightarrow p+\chi+p$ process simply by selecting events with rapidity gaps ${ }^{5}$.

\footnotetext{
${ }^{2}$ See, for example, Ref. [12, and references therein.

${ }^{3}$ In impact parameter $b_{\perp}$ space, the elastic amplitude $A_{\mathrm{el}}\left(b_{\perp}\right)$ arising from the inelastic channels, via unitarity, is given by the square of the inelastic matrix element, $A_{\mathrm{el}}\left(b_{\perp}\right) \propto\left|A_{\text {inel }}\left(b_{\perp}\right)\right|^{2}$, and is therefore concentrated at smaller $b_{\perp}$ where the absorption is stronger; for more details see, for example, [14 and references therein.

${ }^{4}$ For the inclusive process, the momentum transfers $p_{i \perp}$ can be determined by experimentally measuring the transverse energy flows, $E_{i T}$, in the proton fragmentation regions.

${ }^{5}$ As we will discuss below, this is not true for heavy boson production, where we have large Sudakov $T$-factor suppression in order to ensure that the rapidity gaps survive against QCD radiation in the hard subprocess. Since the Sudakov suppression is much stronger for the exclusive amplitude for, say, Higgs production, we have $\sigma_{\text {incl }} \gg \sigma_{\text {excl }}$.
} 


\section{The perturbative QCD approach to double-diffractive $\chi$ produc- tion}

To calculate the cross section for the central exclusive double-diffractive production of $\chi\left(0^{+}\right)$states we use the formalism of Refs. 9, 2, 3. The amplitudes are described by the diagram shown in Fig. 1(a), where the hard subprocess $g g \rightarrow \chi\left(0^{+}\right)$is initiated by gluon-gluon fusion and where the second $t$-channel gluon is needed to screen the colour flow across the rapidity gap intervals. Ignoring, for the moment, the screening corrections of Fig. 1(b), the Born amplitude is of the form [18]

$$
T=A \pi^{2} \int \frac{d^{2} Q_{\perp} P\left(\chi\left(0^{+}\right)\right)}{Q_{\perp}^{2}\left(\vec{Q}_{\perp}-\vec{p}_{1 \perp}\right)^{2}\left(\vec{Q}_{\perp}+\vec{p}_{2 \perp}\right)^{2}} f_{g}\left(x_{1}, x_{1}^{\prime}, Q_{1}^{2}, \mu^{2} ; t_{1}\right) f_{g}\left(x_{2}, x_{2}^{\prime}, Q_{2}^{2}, \mu^{2} ; t_{2}\right),
$$

where the $g g \rightarrow \chi\left(0^{+}\right)$subprocess is specified by $[\underline{3}$

$$
A^{2}=8 \pi \Gamma(\chi \rightarrow g g) / M_{\chi}^{3}
$$

and the polarisation factor ${ }^{6}$

$$
P\left(\chi\left(0^{+}\right)\right)=\left(\vec{Q}_{\perp}-\vec{p}_{1 \perp}\right) \cdot\left(\vec{Q}_{\perp}+\vec{p}_{2 \perp}\right) .
$$

Here $\Gamma(\chi \rightarrow g g)$ is the width including the NLO corrections (that is the $K$ factor). We assume the same NLO correction for the $g g \rightarrow \chi$ vertex as for the $\chi \rightarrow g g$ width, which can be valid only within a certain approximation.

The $f_{g}$ 's in (18) are the skewed unintegrated gluon densities of the proton at the hard scale $\mu$, taken typically to be $M_{\chi} / 2$, with

$$
\begin{aligned}
& Q_{1}=\min \left\{Q_{\perp},\left|\left(\vec{Q}_{\perp}-\vec{p}_{1 \perp}\right)\right|\right\}, \\
& Q_{2}=\min \left\{Q_{\perp},\left|\left(\vec{Q}_{\perp}+\vec{p}_{2 \perp}\right)\right|\right\} .
\end{aligned}
$$

The longitudinal momentum fractions carried by the gluons satisfy

$$
\left(x^{\prime} \sim \frac{Q_{\perp}}{\sqrt{s}}\right) \ll\left(x \sim \frac{M_{\chi}}{\sqrt{s}}\right) \ll 1 .
$$

Below, we assume factorization of the unintegrated distributions,

$$
f_{g}\left(x, x^{\prime}, Q^{2}, \mu^{2} ; t\right)=f_{g}\left(x, x^{\prime}, Q^{2}, \mu^{2}\right) F_{N}(t),
$$

where we parameterize the form factor of the proton vertex by the form $F_{N}(t)=\exp \left(b_{0} t\right)$ with $b_{0}=2 \mathrm{GeV}^{-2}$ [13. In the domain specified by (22) the skewed unintegrated densities are given in terms of the conventional (integrated) densities $g\left(x, Q_{i}^{2}\right)$. To single log accuracy, we have [19] $]^{7}$.

$$
f_{g}\left(x, x^{\prime}, Q_{i}^{2}, \mu^{2}\right)=R_{g} \frac{\partial}{\partial \ln Q_{i}^{2}}\left(\sqrt{T\left(Q_{i}, \mu\right)} x g\left(x, Q_{i}^{2}\right)\right),
$$

where $T$ is the usual Sudakov form factor which ensures that the active gluon does not emit additional real partons in the course of the evolution up to the scale, $\mu$, of the hard process, so that the rapidity gaps survive. This Sudakov factor $T$ is the result of resumming the virtual contributions in the DGLAP evolution. It is given by

$$
T\left(Q_{\perp}, \mu\right)=\exp \left(-\int_{Q_{\perp}^{2}}^{\mu^{2}} \frac{\alpha_{S}\left(k_{t}^{2}\right)}{2 \pi} \frac{d k_{t}^{2}}{k_{t}^{2}} \int_{0}^{1-\Delta}\left[z P_{g g}(z)+\sum_{q} P_{q g}(z)\right] d z\right) .
$$

Here, in analogy to [20], we go beyond the collinear approximation and in the $T$ factor we resum, not just the single collinear logarithms, but the single soft $\log 1 /(1-z)$ terms as well. To a good approximation this can be achieved by taking the upper limit of the $z$ integration in 25) to be

$$
\Delta=\frac{k_{t}}{k_{t}+0.62 M_{\chi}} .
$$

\footnotetext{
${ }^{6}$ For $\chi\left(0^{-}\right)$production the factor $P\left(\chi\left(0^{-}\right)\right)=\left(\left(\vec{Q}_{\perp}-\vec{p}_{1 \perp}\right) \times\left(\vec{Q}_{\perp}+\vec{p}_{2 \perp}\right)\right) \cdot \vec{n}_{0}$, where $\vec{n}_{0}$ is a unit vector in the beam direction; see, for example, [18].

${ }^{7}$ In the actual computations we use a more precise form as given by eq.(26) of Ref. [19].
} 
The square root in (24) arises because the bremsstrahlung survival probability $T$ is only relevant to hard gluons. $R_{g}$ is the ratio of the skewed $x^{\prime} \ll x$ integrated distribution to the conventional diagonal density $g\left(x, Q^{2}\right)$. For $x \ll 1$ it is completely determined [21].

To compare expressions (44) and (the square of) (18), we recall, assuming Regge factorization, that the unintegrated gluon density can be written in the form

$$
f_{g}\left(x, x^{\prime}, Q^{2}, \mu^{2} ; t\right)=R_{g} x^{\lambda\left(Q^{2}, t\right)} \phi\left(Q^{2}, \mu^{2}\right) g_{N}(t),
$$

These factors $x_{i}^{\lambda}$, in the $f_{g}$ 's in (18), play the role of the Regge factors $x_{i}^{1-\alpha_{P}\left(t_{i}\right)}$ in (4). Moreover the form factor $F_{N}(t)$ in (23) describes the $t$ dependence of the coupling $g_{N}(t)$, while the remainder of the integral in (18) gives the product $V g_{N}^{2}(0)$.

\section{The cross section for exclusive diffractive $\chi$ production}

The uncertainties in the perturbative QCD predictions for the exclusive double-diffractive production of heavy (Higgs) states of mass $120 \mathrm{GeV}$ were discussed in Ref. [20. The uncertainties come from the infrared contribution to the $Q_{\perp}$ integral, (18), from the choice of factorization scale, from the lack of precise knowledge of the gluon at low scales and small $x$, from deviations from formula (24) for large-angle gluon emission and from the uncertainty in the calculation of the screening correction, $\hat{S}^{2}$. For a Higgs $0^{+}$state the total uncertainty was estimated to be a factor of almost 2.5, that is up to almost 2.5, and down to almost $1 / 2.5$ the quoted value. In Ref. 20 it was also estimated that the uncertainties for producing a heavy $0^{-}$state would be larger by almost another factor of 2.5 .

For the exclusive double-diffractive production of light $\chi$ states the infrared uncertainties will be even larger. We computed the integral in (18) using the GRV94 parametrization [22]- the only available parton set which extends down to a very low scale $\left(Q^{2} \simeq 0.4 \mathrm{GeV}^{2}\right)$. To be safe, we choose a rather low energy, $\sqrt{s}=60 \mathrm{GeV}$, which corresponds to $x \sim 0.05$, where the GRV, MRST, CTEQ gluons are approximately equal; also where the $p p$ total cross section is still approximately flat, which provides a simple normalization of the Pomeron-nucleon vertex. We find that half of the contribution to the amplitude (18) comes from the region $Q_{\perp}<0.85 \mathrm{GeV}$. Clearly perturbative calculations are not justified in this domain. For low $Q_{\perp}$ the gluon propagator will be modified by non-perturbative dynamics; for example by the presence of $G_{\mu \nu}^{a} G_{\mu \nu}^{a}$ condensates, effective gluon masses, confinement forces etc. It appears that these dynamics effectively suppress the low $Q_{\perp}$ contribution; for recent reviews see, for example, Ref. [23. Therefore the predictions below should be regarded as just an order-of-magnitude indication of the expected rates. If we evaluate amplitude (18) using GRV94HO partons 22 then we find, for $\sqrt{s}=60 \mathrm{GeV}$, that the 'bare' cross section is

$$
\left.\frac{d \sigma}{d p_{1 \perp}^{2} d p_{2 \perp}^{2} d y}\right|_{p_{1 \perp}=p_{2 \perp}=0, y=0} \simeq 8 \mu \mathrm{b} / \mathrm{GeV}^{4}
$$

whereas if the contribution from the region $Q_{\perp}<0.85 \mathrm{GeV}$ is neglected then we obtain $2 \mu \mathrm{b} / \mathrm{GeV}^{4}$. By the bare cross section we mean the prediction before including the rapidity gap survival factor $\hat{S}^{2}$.

We emphasize that the diagram of Fig. 1(a) plays the dominant role when the transverse size of the Pomeron (that is the transverse separation of the $x$ and $x^{\prime}$ gluons) is much larger than the size of the produced boson. This is the case for Higgs production ${ }^{8}$, but it is not so clear for $\chi_{c}$ production. The other extreme is to assume that the transverse size of the Pomeron is relatively small, so that the Pomeron couples to each individual quark. This extreme corresponds to the 'additive quark model'. In this case the fusion vertex may be calculated from the Feyman diagram shown in Fig. 3. The coupling of the charm quarks to form the $\chi_{c}$ meson is described in terms of the $\chi_{c}$ meson wave function, $\psi(r)$, for which we apply the standard non-relativistic formalism, see, for example, Refs. [25, 26].

Next we have to estimate the coupling $g_{c}$ of the charm quark to the Pomeron. For this, we can use the analysis of the data for charmonia production on nuclei [27]. The analysis [28] showed that the cross section for

\footnotetext{
${ }^{8}$ Indeed it has been shown [24 that there is a negligible contribution from graphs where both gluons (forming the Pomeron) couple to the top-quark loop.
} 


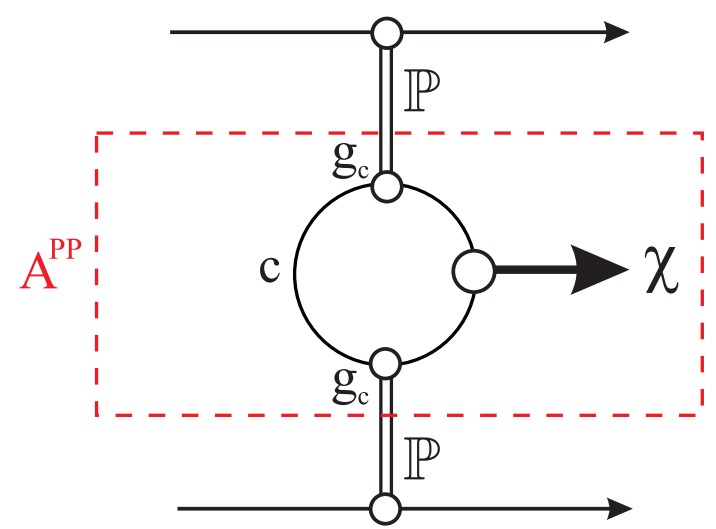

Figure 3: The diagram used to calculate the non-perturbative contribution to the exclusive process $p p \rightarrow p+\chi+p$. The amplitude, $A^{P P}$, describing the Pomeron-Pomeron $\rightarrow \chi$ subprocess, is controlled by the charm quark loop. It is evaluated using (32).

$J / \psi$ on protons can be parametrised as $^{9}$

$$
\sigma=\sigma_{0}\left(s / s_{0}\right)^{\Delta}
$$

with $\Delta=0.21$ and $\sigma_{0}=3.6 \mathrm{mb}$, for $s_{0}=100 \mathrm{GeV}^{2}$. Thus the additive quark model estimate of the charm quark-proton cross section ${ }^{10}$ is $\sigma(c p) \simeq 2 \mathrm{mb}$, for $s_{\psi p} \simeq 200 \mathrm{GeV}^{2}$, which corresponds to $\sqrt{s}=60 \mathrm{GeV}$ of (28). Phenomenologically, the Pomeron-quark coupling may have a scalar or Dirac $\gamma_{\mu}$ matrix form. The latter means that Pomeron exchange is similar to photon exchange. Thus it is reasonable to estimate the $P P-\chi$ coupling using the known $\chi \rightarrow \gamma \gamma$ decay width, $\Gamma\left(\chi\left(0^{+}\right) \rightarrow \gamma \gamma\right) \simeq 3 \mathrm{keV}$ [30]. However the analogy between the Pomeron and the photon cannot be exact. First, the Pomeron has even $C$-parity. Moreover the Pomeron is not a gauge field and so its $\gamma_{\mu}$ vertex would not correspond to a conserved current [31. Indeed, unlike $\chi \rightarrow \gamma \gamma$, for $P P \rightarrow \chi$ there are no graphs with a permutation of the two Pomeron vertices. The reason is that in Regge theory all interactions are strongly ordered in rapidity space. Thus we omit the second term in eq.(2) of Ref. [26].

If we follow the above procedure, then, for $\chi_{c}\left(0^{+}\right)$, we find that the $P P \rightarrow \chi$ vertex, $V$ of (4), is

$$
V \equiv A^{P P}=g_{c}^{2} \frac{a}{\sqrt{6}} \frac{4}{m_{c}},
$$

whereas the $\chi \rightarrow \gamma \gamma$ decay amplitude is

$$
A^{\gamma \gamma}=\frac{4}{9}\left(4 \pi \alpha_{Q E D}\right) \frac{a}{\sqrt{6}} \frac{12}{m_{c}},
$$

where $g_{c}$ is the Pomeron-c quark coupling, and $a=\sqrt{3 / 4 \pi m_{c}} \psi^{\prime}(0)$, where $\psi^{\prime}(0)$ is the value of the first derivative of the bound-state wave function at the origin. In this way we can normalize the $P P \rightarrow \chi$ vertex in terms of the known $\chi \rightarrow \gamma \gamma$ decay width. We obtain

$$
A^{P P}=\frac{3 g_{c}^{2}}{16 \pi \alpha_{Q E D}} A^{\gamma \gamma}
$$

If we were to choose a scalar Pomeron-quark coupling, then we would obtain an $A_{P P}$ amplitude which is a factor $5 / 4$ larger. However we prefer the $\gamma_{\mu}$ form of the vertex, which conserves the $s$-channel helicity of the quark.

If, now, we take $\sigma(c p)=2 \mathrm{mb}$, and normalize the $A^{P P}$ amplitude as in (32), then we find that the non-

\footnotetext{
${ }^{9}$ The values of the cross section are in qualitative agreement with the analysis of Ref. [29].

${ }^{10}$ Note, however, a similar analysis of $\psi^{\prime}$ gives a somewhat larger value of $\sigma(c p)$.
} 
perturbative contribution ${ }^{11}$ to exclusive double-diffractive $\chi_{c}$ cross section is

$$
\left.\frac{d \sigma^{\text {nonpert }}}{d p_{1 \perp}^{2} d p_{2 \perp}^{2} d y}\right|_{p_{1 \perp}=p_{2 \perp}=0, y=0} \simeq 1.1 \mu \mathrm{b} / \mathrm{GeV}^{4} \times \hat{S}^{2},
$$

with $t_{i} \simeq-p_{i \perp}^{2}$. Note that the contribution of Fig. 3 is real (if we assume $\operatorname{Re} / \operatorname{Im} \ll 1$ ), and therefore does not interfere with the imaginary amplitude of Fig.1. Thus we simply add these two contributions to the cross section. To be conservative, we only include the perturbative contribution of gluons with $Q_{\perp}>0.85 \mathrm{GeV}$ : that is

$$
\left.\frac{d \sigma^{\text {pert }}}{d p_{1 \perp}^{2} d p_{2 \perp}^{2} d y}\right|_{p_{1 \perp}=p_{2 \perp}=0, y=0} \simeq 2 \mu \mathrm{b} / \mathrm{GeV}^{4} \times \hat{S}^{2},
$$

at $\sqrt{s}=60 \mathrm{GeV}$; see the discussion below (28).

Now we need to consider the energy dependence. First we note that the data for $J / \psi$ photoproduction [15] are described by a Pomeron with $\alpha_{P}(0)-1 \simeq 0.2$. The $c \bar{c}$ system in this process has a similar mass to the subprocess we are concerned with and so we take $\alpha_{P}(0)-1=\Delta=0.2$. The same value of $\Delta$ describes the behaviour of the $J / \psi$-proton cross section, and is also consistent with the small $x$ behaviour found in the global parton analyses of deep inelastic scattering data. Adding together the above perturbative and non-perturbative contributions at $\sqrt{s}=60 \mathrm{GeV}$, and using the appropriate energy dependence, $s^{2 \Delta}$ of (10), we find the cross section for $p p \rightarrow p+\chi_{c}+p$, at the Tevatron energy, $\sqrt{s}=2 \mathrm{TeV}$, is

$$
\left.\frac{d \sigma\left(\chi_{c}\right)}{d y}\right|_{y=0}=130 \mathrm{nb}
$$

Here we have included the survival factor $\hat{S}^{2}=0.07$ of the rapidity gaps, (13), and integrated over the transverse momenta $p_{i \perp}$. To obtain an approximate expression for the $t$ dependence of the exclusive double-diffractive cross section, we neglect the possible difference in the form factors corresponding to Figs. 1 and 3 , and the effects of the screening corrections on the $p_{i \perp}$ distributions, which results in the behaviour $F_{N}\left(t_{1}\right)^{2} F_{N}\left(t_{2}\right)^{2} \exp \left(\alpha^{\prime}\left(t_{1}+\right.\right.$ $\left.\left.t_{2}\right) \ln \left(s / M_{\chi}^{2}\right)\right)$.

To obtain the total observable cross section at the Tevatron we integrate over an effective rapidity interval of $\Delta y=5$ which gives

$$
\sigma_{\text {excl }}\left(\chi_{c}\right)=650 \mathrm{nb} .
$$

This, more detailed estimate, is very close to our previous result [2].

Note that the production of a (non-relativistic) $2^{++}$quarkonium state may allow the discrimination between the two mechanisms shown in Fig. 1 and Fig. 3. As we already discussed (see Refs. 20 [5] for more details) in the on-shell approximation the $J_{z}(g g)=0$ amplitude vanishes. Then, the only contribution comes from the amplitude for $\left|J_{z}\right|=2$ quarkonium $\left(2^{++}\right)$production, arising from the violation of the $J_{z}=0$ selection rule due to non-zero $p_{\perp}$ of the protons. Thus, we expect that the amplitude corresponding to Fig. 1 vanishes for forward $2^{++}$quarkonium production. On the other hand there is no selection rule similar to $J_{z}(g g)=0$ for the general amplitude of Fig. 2 (though even $\mathrm{P}$ and $\mathrm{C}$ still hold). In particular, the amplitude of Fig. 3 allows $2^{++}$production at any $p_{\perp}$ and, therefore, does not vanish as $p_{\perp} \rightarrow 0$. Another difference between the contributions of Fig. 1 and Fig. 3, is that, in Fig. 3, a centrally-produced $\chi\left(2^{++}\right)$meson has $J_{z}=0$, while for Fig. 1 non-relativistic $2^{++}$quarkonium has $\left|J_{z}\right|=2$.

For completeness we gather together all our predictions for the cross sections for double-diffractive $\chi$ production in Table 1. The evaluation of the inclusive cross section is discussed in the next Section, and also in Section 8. Comments on $\chi_{b}$ may be found in Section 6 . Although the cross sections are large, we note that the relevant $\chi$ decay branching fractions are small. Traditionally, the experimentally favoured decay is $\chi_{c} \rightarrow \gamma J / \psi \rightarrow \gamma \mu^{+} \mu^{-}$ with branching fraction $6 \times 10^{-4}$. On the other hand, $\chi_{c} \rightarrow\left(\pi^{+} \pi^{-}+K^{+} K^{-}\right)$has branching fraction 0.011 , and this 2 -prong configuration could prove more favourable. For $\chi_{b}$ the corresponding branching ratios are not so well known, but it hard to imagine that the situation will be better. Recall that $\chi_{b}$ decays dominantly into two gluon jets. Unfortunately this decay channel will suffer from a large background from QCD dijet production in

\footnotetext{
${ }^{11}$ We call it "non-perturbative" although, strictly speaking, the true underlying dynamics of the $\sigma(c p)$ interaction is not well established.
} 


\begin{tabular}{|c|cc|cc|}
\hline & \multicolumn{2}{|c|}{ Tevatron $\sqrt{s}=2 \mathrm{TeV}$} & \multicolumn{2}{c|}{$\mathrm{LHC} \sqrt{s}=14 \mathrm{TeV}$} \\
& $\chi_{c}$ & $\chi_{b}$ & $\chi_{c}$ & $\chi_{b}$ \\
\hline$d \sigma_{\text {excl }} /\left.d y\right|_{y=0}$ & 130 & 0.2 & 340 & 0.6 \\
$\sigma_{\text {excl }}$ & 650 & 0.5 & 3000 & 4 \\
\hline$d \sigma_{\text {incl }} /\left.d y\right|_{y=0}$ & 13 & 0.06 & 30 & 0.2 \\
$\sigma_{\text {incl }}$ & 70 & 0.3 & 200 & 2 \\
\hline
\end{tabular}

Table 1: The cross sections (in nb) for exclusive and inclusive double-diffractive $\chi_{c}$ and $\chi_{b}$ production at the Tevatron and LHC. From the discussion in the text, we see that these predictions should be regarded as order of magnitude estimates. The accuracy is limited because of the low scale associated with $\chi$ production.

the same kinematical configuration. Using the results of Ref. [3], and neglecting the K-factor in the background calculation, we estimate the signal-to-background ratio to be $S / B \sim 5 \times 10^{-3} / \Delta M(\mathrm{GeV})$. Certainly we would expect that there is a sizeable (on the $\sim 1 \%$ level) branching ratio for $\chi_{b}\left(0^{+}\right) \rightarrow \gamma+\Upsilon$ decay. But at the moment there is no experimental data on this channel. Therefore the situation regarding detection of the $\chi_{b}\left(0^{+}\right)$in the central detector remains unclear.

What are the uncertainties in our cross-section predictions? First, it is interesting to note that the results are almost independent on the choice of the slope, $b=2 b_{0}+2 \alpha^{\prime} \ln 1 / x$, of the $p_{i \perp}$ distributions. The reduction caused by a larger slope is largely compensated by an increased survival factor $\hat{S}^{2}$ for the more peripheral reaction. For example, varying the slope $2 b_{0}$ from 4 to $6 \mathrm{GeV}^{-2}$ changes the individual values of $\hat{S}^{2}$ and $b^{2}$ by approximately a factor of 2 , but decreases $\hat{S}^{2} / b^{2}$ by only about $10-15 \%$ at the Tevatron and the LHC energies ${ }^{12}$. On the other hand, the cross section would be 4 times smaller if we were to take a 'soft' Pomeron intercept, $\alpha_{P}(0)-1=\Delta=0.1$, instead of 0.2 , for the extrapolation from $\sqrt{s}=60 \mathrm{GeV}$ to the Tevatron energy. Moreover the charm quark cross section may be larger (see the footnote below (29)), and there may be an additional contribution from the low $Q_{\perp}$ domain, $Q_{\perp}<0.85 \mathrm{GeV}$, of the gluon-induced amplitude (18). Thus the cross section estimate of (36) could be a factor of 4 higher.

\section{The rates for inclusive double-diffractive $\chi$ production}

The cross section for double-diffractive inclusive production is calculated is an analogous way to the exclusive one. Indeed, it may be considered as 'exclusive' $\chi$ meson production in parton-parton collisions. The flux of initial partons is given by the known parton distributions inside a proton, while the unintegrated skewed distributions (inside an individual) parton, $f_{g}$, are calculated in terms of the non-forward BFKL amplitudes. It is important to note that here there is no form factor for point-like parton scattering and that there are sizable contributions over a much larger range of transverse momenta $p_{i \perp}$, with $i=1,2$. Therefore we keep just the leading-order contributions; that is, the integrals over $Q_{\perp}, p_{1 \perp}$ and $p_{2 \perp}$ take logarithmic forms. In particular

$$
\frac{d^{2} Q_{\perp} P\left(\chi\left(0^{+}\right)\right)}{Q_{\perp}^{2}\left(\vec{Q}_{\perp}-\vec{p}_{1 \perp}\right)^{2}\left(\vec{Q}_{\perp}+\vec{p}_{2 \perp}\right)^{2}} \text { takes the form } \frac{d^{2} Q_{\perp}}{Q_{\perp}^{2}} \frac{\vec{p}_{1 \perp} \cdot \vec{p}_{2 \perp}}{p_{1 \perp}^{2} p_{2 \perp}^{2}}
$$

so that the final integrals in the expression for the cross section become

$$
\frac{d^{2} Q_{\perp}}{Q_{\perp}^{2}} \frac{d^{2} Q_{\perp}^{\prime}}{Q_{\perp}^{\prime 2}} \frac{d^{2} p_{1 \perp}}{p_{1 \perp}^{2}} \frac{d^{2} p_{2 \perp}}{p_{2 \perp}^{2}}
$$

see Refs. [32, 3] for details.

As mentioned before, for a heavy centrally produced state, such as a Higgs boson, the cross section for inclusive double diffractive production is predicted to be much larger than that for exclusive production. The reasons are as follows:

\footnotetext{
${ }^{12}$ For the same reason the cross section predictions for exclusive double-diffractive Higgs production are practically independent of the value taken for the slope $b$.
} 
(a) due to the absence of proton form factors in the inclusive process there are sizeable contributions to the $d p_{\perp}^{2} / p_{\perp}^{2}$ integral with $Q_{\perp}<p_{\perp}<M_{H} / 2$,

(b) the $T$-factor suppression is weaker for inclusive production, as now $T\left(p_{\perp}, M_{H}\right)>T\left(Q_{\perp}, M_{H}\right)$,

(c) for inclusive production there is no P-even $J_{z}=0$ selection rule [33, 34, 2, 3, which suppresses ${ }^{13}$, for example, $0^{-}, 2^{+}$(non-relativistic) quarkonium exclusive production [2, 5, 14].

On the other hand for the relatively light $\chi$ states the typical values of $p_{\perp}$ transferred through the Pomeron, and $Q_{\perp}$ of the internal gluon (inside the Pomeron) are of the same order. As a consequence inclusive production is not strongly enhanced in comparison with exclusive production. Insight into this result can be obtained from the discussion leading to (17). It is therefore not surprising that, using the perturbative QCD approach of the previous section, we estimate that

$$
\sigma_{\text {incl }}\left(\chi_{c}\left(0^{+}\right)\right) \simeq 70 \mathrm{nb}
$$

at the Tevatron energy $\sqrt{s}=2 \mathrm{TeV}$, which is about 10 times less than the exclusive double-diffractive cross section $\sigma_{\text {excl }} \simeq 650 \mathrm{nb}$. It is interesting to note that the perturbative QCD estimate reflects the smallness of the triple-Pomeron vertex. The low QCD estimate arises from the small gluon density (at low $x$ and small scale) obtained in the global parton analyses of deep-inelastic and related data. As we have already mentioned, recently the CDF collaboration [10] have reported their first results on $J / \psi+\gamma$ central production, with rapidity gaps on either side, and with a mass consistent with that of $\chi_{c}$. These results are still preliminary, but the observed cross section is compatible with the expectations of Ref. [2], assuming that the background issues are well understood and turn out to be small. With more data to come, and with a study of the background, it will be possible to obtain more detailed information on this process.

In going from the Tevatron to the LHC energy, $\sqrt{s}=14 \mathrm{TeV}$, the double-diffractive inclusive cross section (for a fixed rapidity gap interval), $\sigma_{\text {incl }}$, is expected to grow as $s^{\Delta}$, with $\Delta \simeq 0.2$. Now we have to integrate over the rapidity gap intervals $\Delta \eta>3$, that is over the masses $d M_{1}^{2} / M_{1}^{2}$ and $d M_{2}^{2} / M_{2}^{2}$. In the limit of small fixed masses $M_{1}, M_{2}$ the integrand grows faster - as $s^{2 \Delta}$ - similar to the elastic cross section. On the other hand the rapidity gap survival factor $\hat{S}^{2}$ decreases as $s^{-0.1}[13$, see [15]. To be explicit, we find [13.

$$
\hat{S}^{2}(\mathrm{LHC}) / \hat{S}^{2} \text { (Tevatron) } \simeq 2 / 3 .
$$

We see that the energy dependence of the cross section and the behaviour of the survival factor partially compensate each other, so

$$
\frac{d \sigma_{\text {incl }}}{d y}(\text { LHC }) \simeq 2.5 \frac{d \sigma_{\text {incl }}}{d y} \text { (Tevatron) }
$$

for $y=0$. At the LHC, a larger rapidity interval is available, $\Delta y \sim 8$, which leads to an increased cross section

$$
\sigma_{\text {incl }}\left(\chi_{c}\left(0^{+}\right)\right) \simeq 200 \mathrm{nb} .
$$

For the exclusive process, the cross section $d \sigma / d y$ is proportional to $s^{2 \Delta}$. In fact the QCD estimate gives $\sigma_{\text {excl }}\left(\chi_{c}\left(0^{+}\right)\right) \simeq 3000 \mathrm{nb}$ at the LHC. These predictions are collected together in Table 1 .

\section{Predictions for double-diffractive $\chi_{b}$ production}

The perturbative QCD predictions of the cross sections of double-diffractive $\chi_{b}$ production are more justified than those for $\chi_{c}$, on account of the larger $\chi_{b}$ mass. For this reason we only evaluate the perturbative amplitude (18), which corresponds to the diagrams of Fig.1. Moreover, as before, we keep just the contribution from the $Q_{\perp}>0.85 \mathrm{GeV}$ integration region. This is found to give about $\frac{2}{3}$ of the total amplitude (18) obtained using GRV94HO partons 22]. The energy dependence of the bare cross section is driven by the small $x$ behaviour of the gluon. However we use again $\Delta=0.2$, which is much more consistent with the recent partons sets (for example [36]) than the GRV94 set.

Another uncertainty is the absence of an experimental measurement of the gluonic width, $\Gamma\left(\chi_{b}\left(0^{+}\right) \rightarrow g g\right)$. Potential models 37] predict a bare width of $\Gamma_{0}\left(\chi_{b} \rightarrow g g\right)=1.3-1.9 \mathrm{MeV}$, which should be multiplied by the

\footnotetext{
${ }^{13}$ The exclusive double-diffractive production of vector and axial vector states is suppressed as a consequence of the Landau-Yang theorem [35] for massless gluons.
} 
NLO correction factor $K=\left(1+9.8 \alpha_{S} / \pi\right)$ [38. This is some 5 times larger than the prediction $\Gamma_{0}\left(\chi_{b} \rightarrow g g\right) \simeq 350$ $\mathrm{keV}$ obtained from QCD lattice calculations [39]. For the predictions of the cross sections of double-diffractive $\chi_{b}$ production at the Tevatron and LHC, which we show in Table 1, we have taken $\Gamma\left(\chi_{b} \rightarrow g g\right)=1.3 \mathrm{~K} \mathrm{MeV}$. Note that these predictions are larger than those given in Ref. 2], where the lattice estimate of the width was used $^{14}$; see also Ref. [5].

\section{Implications of the larger exclusive rate for $\chi_{c}$ production}

The QCD predictions of the double-diffractive exclusive and inclusive $\chi_{c}$ production show that the former process dominates. Indeed at the LHC we estimate

$$
\sigma_{\text {excl }} / \sigma_{\text {incl }} \sim 10
$$

It means that by just selecting events with two rapidity gaps we will observe mainly the exclusive process, even without requiring the forward protons to be tagged!

One topical application would be to search for the exclusive production of the new $X(3872)$ charmonium state observed recently by the Belle and CDFII collaborations [42]. If this state were a radial excitation of the $\chi_{c}\left(0^{+}\right)$meson, then it would be observed with a cross section

$$
\sigma(p p \rightarrow p+X+p) \sim 100(500) \mathrm{nb}
$$

at the Tevatron (LHC). Of course, looking for a specific decay $X \rightarrow \psi \pi^{+} \pi^{-} \rightarrow \mu^{+} \mu^{-} \pi^{+} \pi^{-}$will reduce the signal by about 1000 , but still the cross section is large.

Since the $X$ production rate is proportional to the $X \rightarrow g g$ decay width, a measurement of the $p p \rightarrow p+X+p$ cross section can give an estimate of the $X \rightarrow g g$ coupling. To reduce the (large) theoretical uncertainties it would be better to compare the rates for $X(3872)$ and $\chi_{c}(3415)$, as the $g g$ width is known for the latter state ${ }^{15}$.

If $X(3872)$ were a $D \bar{D}^{*}$-molecule (see, for instance, 43]), then the exclusive signal would probably not be seen ${ }^{16}$. Also note that, due to the P-even, $J_{z}=0$ selection rule ${ }^{17}$, the exclusive cross section is suppressed for the double-diffractive production of $0^{-}, 2^{+}$or C-odd bosons. Thus by observing the $X(3872)$ state in the exclusive process we would conclude that it is a C-even, and most probably a $0^{+}$, particle.

\section{Parity determination}

With sufficient luminosity we can use the inclusive process, (2), to study $0^{-}$or $2^{++}$production by PomeronPomeron fusion. If the transverse momentum $p_{\perp}$ transferred through the Pomeron is small, $p_{\perp} \ll Q_{\perp}$, then the inclusive process is controlled by the same P-even, $J_{z}=0$ selection rule as exclusive production. However if we select events with large $p_{i \perp}$ then the production of P-odd and $J_{z}= \pm 2$ states become possible. Note that large $p_{\perp}$ 's can be measured as the transverse energy flows, $p_{1 \perp}=-E_{1 \perp}$ and $p_{2 \perp}=E_{2 \perp}$, in the proton fragmentation regions. Moreover the major part of the energy flow is carried by one jet (with lowest rapidity $|y|$ in the centre-of-mass frame). Indeed at lowest order this jet carries the entire $E_{i \perp}$. These measurements allow a study of the $\phi$ dependence, where $\phi$ is the azimuthal angle between the energy flows $\vec{E}_{1 \perp}$ and $\vec{E}_{2 \perp}$. The $\phi$ distribution depends on the parity of the produced system [20]:

$$
d \sigma / d \phi \propto 1+\cos 2 \phi \quad \text { for a natural parity state, } P=(-1)^{J}
$$

\footnotetext{
${ }^{14}$ Note, however, that the calculations of the matrix elements in Ref. [39] were based on the lattice NRQCD results of Ref. [40, which used quenched approximation. As was shown in 41, this approximation underestimates the NRQCD matrix elements. Thus the values of the widths, in the case of three light quark flavours, should be higher than the quenched results.

${ }^{15}$ The effective $g g^{P P}$ luminosity, $\mathcal{L}\left(M^{2}\right)$, is essentially flat in this mass interval.

${ }^{16}$ In this case there should be some contribution from the $D$-meson loop, analogous to the charm quark loop in Fig. 3 , but it is expected to be small.

${ }^{17}$ Unfortunately the $J_{z}=0$ selection rule is less precise at these low scales. The admixture of $\left|J_{z}\right|=2$ states may be up to $20-30 \%$. However, it is encouraging that expectations based on this rule appear to be in good qualitative agreement with the available data on double-diffractive meson production; see [14] for details.
} 


\begin{tabular}{|lccc|}
\hline & $E_{\perp}>3$ & $E_{\perp}>5$ & $E_{\perp}>7$ \\
\hline Tevatron & & & \\
$\sigma\left(\chi_{c}\right)$ & 500 & 20 & \\
$\sigma\left(\chi_{b}\right)$ & 35 & 5 & \\
\hline LHC & & & \\
$\sigma\left(\chi_{c}\right)$ & 6000 & 400 & 40 \\
$\sigma\left(\chi_{b}\right)$ & 500 & 100 & 30 \\
\hline
\end{tabular}

Table 2: The inclusive double-diffractive cross sections (in $\mathrm{pb}$ ) for various choices of the cut on the transverse energy flows, $E_{i \perp}$ (in $\mathrm{GeV}$ ). To account for the large gluon virtuality, which occurs for large $E_{i \perp}$, we follow a procedure similar to that used in Ref. [26] for the calculation of the amplitude for the decay of $\chi\left(0^{+}\right)$into virtual photons.

whereas

$$
d \sigma / d \phi \propto 1-\cos 2 \phi \quad \text { for an unnatural parity state, } P=-(-1)^{J} .
$$

We emphasize, that since we consider rather large $E_{\perp}$, these angular distributions are less sensitive to soft rescattering.

To get some idea of the event rates, we present the cross sections for $p p \rightarrow X+\chi\left(0^{+}\right)+Y$ in Table 2, for different choices of the $E_{\perp}$ cut. If we take $E_{i \perp}>3 \mathrm{GeV}$ at the Tevatron, then $\sigma_{\text {incl }}\left(\chi_{c}\left(0^{+}\right)\right) \sim 0.5 \mathrm{nb}$, whereas for $E_{i \perp}>7 \mathrm{GeV}$ at the LHC we estimate $\sigma_{\text {incl }}\left(\chi_{c}\left(0^{+}\right)\right) \sim 40 \mathrm{pb}$. Note that the reliability of the predictions with large $E_{\perp}$ cuts is better, since we do not enter the infrared domain. These are rather large cross sections, but recall that the relevant decay branching fractions are small, see Section 4.

Finally, note that the ratio of the $0^{++}$and $2^{++}$production cross sections is

$$
\frac{\sigma_{\text {incl }}\left(2^{++}\right)}{\sigma_{\text {incl }}\left(0^{++}\right)}=\frac{5}{4} \frac{\Gamma\left(2^{++}\right)}{\Gamma\left(0^{++}\right)}
$$

where we have neglected the mass difference between the $M\left(2^{++}\right)$and $M\left(0^{++}\right)$mesons.

\section{Conclusions}

We find that both the Regge formalism and perturbative QCD predict essentially the same qualitative behaviour for the central double-diffractive production of 'heavy' $\chi_{c}\left(0^{++}\right)$and $\chi_{b}\left(0^{++}\right)$mesons. Due to the low scale, $M_{\chi} / 2$, there is a relatively small contribution coming from the process in which the incoming protons dissociate. Therefore simply selecting events with a rapidity gap on either side of the $\chi$, almost ensures that they will come from the exclusive reaction, $p p \rightarrow p+\chi+p$.

We evaluated the expected double-diffractive cross sections and demonstrated that they are sufficiently large for both $\chi_{c}$ and $\chi_{b}$ meson production to be observed. Since the rapidity-gap survival factor, $\hat{S}^{2}$, decreases with energy, the cross section $d \sigma / d y$ at the LHC energy only exceeds that at the Tevatron energy by a factor of about 3. Our cross section predictions should be regarded as only order of magnitude estimates, because of the low scale, nevertheless these processes can be very informative. For example, the observation of the new charmonium state $X(3872)$ in the exclusive process $p p \rightarrow p+X+p$ would be a strong argument in favour of its quantum numbers being $J^{P C}=0^{++}$. Moreover, since the exclusive cross section is proportional to the gluonic width, it will be possible to measure the width $\Gamma(X \rightarrow g g)$ by comparing the rates of exclusive $\chi_{c}$ and $X$ production. While there is a sizeable uncertainty in the predictions for the overall rates of double difffractive $\chi$ production, we stress that the kinematic distributions, for example the $\chi$ transverse momentum distributions, should be more reliable.

Although exclusive $\chi$ production is expected to dominate, the event rates should be large enough to select double-diffractive dissociative events with large transverse energy flows in the proton fragmentation regions. Such events are particularly interesting. First, in this case, the large value of $E_{\perp}$ provides the scale to justify 
the validity, and the reasonable accuracy, of the perturbative QCD calculation of the cross section. Next, by measuring the azimuthal distribution between the two $E_{\perp}$ flows we can determine the parity of the centrally produced system.

An interesting extension of the exclusive double-diffractive approach, would be to observe central open $b \bar{b}$ production; namely $b, \bar{b}$ jets with $p_{\perp} \gtrsim m_{b}$. Again, this would put the application of perturbative QCD on a sounder footing. It would allow a check of the perturbative formalism, as well as a study of the dynamics of $b \bar{b}$ production.

\section{Acknowledgements}

We thank Mike Albrow, Albert De Roeck, Risto Orava and Angela Wyatt for useful discussions. ADM thanks the Leverhulme Trust for an Emeritus Fellowship and MGR thanks the IPPP at the University of Durham for hospitality. This work was supported by the UK Particle Physics and Astronomy Research Council, by grant RFBR 04-02-16073 and by the Federal Program of the Russian Ministry of Industry, Science and Technology SS-1124.2003.2.

\section{References}

[1] D. Robson, Nucl. Phys. B130 (1977) 328;

F.E. Close, Rept. Prog. Phys. 51 (1988) 833.

[2] V.A. Khoze, A.D. Martin and M.G. Ryskin, Eur. Phys. J. C19 (2001) 477, erratum C20 (2001) 599.

[3] V.A. Khoze, A.D. Martin and M.G. Ryskin, Eur. Phys. J. C23 (2002) 311.

[4] J. Pumplin, Phys. Rev. D47 (1993) 4820.

[5] Feng Yuan, Phys. Lett. B510 (2001) 155.

[6] see, for example, M. Cacciari, S. Frixione, M.L. Mangano, P. Nason and G. Ridolfi, arXiv:hep-ph/0312132, and references therein.

[7] M. Krämer, Prog. Part. Nucl. Phys. 47 (2001) 141;

A. Pineda, Nucl. Phys. Proc. Suppl. 93 (2001) 188;

A. Hoang, arXiv: hep-ph/0204299

G.T. Bodwin, arXiv: hep-ph/0212203

A. Vairo, arXiv:hep-ph/0311303.

[8] A. De Roeck, V.A. Khoze, A.D. Martin, R. Orava and M.G. Ryskin, Eur. Phys. J. C25 (2002) 391.

[9] V.A. Khoze, A.D. Martin and M.G. Ryskin, Eur. Phys. J. C14 (2000) 525.

[10] M. Gallinaro (representing the CDF Collaboration), FERMILAB-CONF-03-403-E, Nov.2003, arXiv:hep-ph/0311192.

[11] TOTEM collaboration: TDR, CERN-LHCC-2004-002, TOTEM-TDR-001, 7th. Jan. 2004.

[12] A.B. Kaidalov, V.A. Khoze, A.D. Martin and M.G. Ryskin, Eur. Phys. J. C21 (2001) 521.

[13] V.A. Khoze, A.D. Martin and M.G. Ryskin, Eur. Phys. J. C18 (2000) 167.

[14] V.A. Khoze, A.D. Martin and M.G. Ryskin, Eur. Phys. J. C24 (2002) 581.

[15] ZEUS Collaboration: S. Chekanov et al., Eur. Phys. J. C24 (2002) 345.

[16] A.B. Kaidalov, V.A. Khoze, Yu.F. Pirogov and N.L. Ter-Issakyan, Phys. Lett. B45 (1973) 493;

A.B. Kaidalov and K.A. Ter-Martirosyan, Nucl. Phys. B75 (1974) 471. 
[17] A.B. Kaidalov, Phys. Rept. 50 (1979) 157.

[18] A.B. Kaidalov, V.A. Khoze, A.D. Martin and M.G. Ryskin, Eur. Phys. J. C31 (2003) 387.

[19] A.D. Martin and M.G. Ryskin, Phys. Rev. D64 (2001) 094017.

[20] A.B. Kaidalov, V.A. Khoze, A.D. Martin and M.G. Ryskin, Eur. Phys. J. C33 (2004) 261.

[21] A.G. Shuvaev, K.J. Golec-Biernat, A.D. Martin and M.G. Ryskin, Phys. Rev. D60 (1999) 014015.

[22] M. Glück, E. Reya and A. Vogt, Z. Phys. C67 (1995) 43.

[23] C.D. Roberts and S.M. Schmidt, Prog. Part. Nucl. Phys. 45 (2000) S1;

R. Alkofer and L. von Smekal, Phys. Rept. 353 (2001) 281;

R. Alkofer and C.S. Fischer, arXiv:hep-ph/0309089, and references therein.

[24] H.J. Lu and J. Milana, Phys. Rev. D51 (1995) 6107.

[25] R. Barbieri, R. Gatto and R. Kögerler, Phys. Lett. 60B (1975) 183.

[26] J.H. Kühn, J. Kaplan and E.G.O. Safiani, Nucl. Phys. 157 (1979) 125.

[27] R.L. Anderson et al., Phys. Rev. Lett. 38 (1977) 263;

NA50 Collaboration: B. Alessandro et al., Phys. Lett. B553 (2003) 167;

FNAL E866/NuSea collaboration: M.J. Leitch et al., Phys. Rev. Lett. 84 (2000) 3256.

[28] J. Hüfner, Yu.P. Ivanov, B.Z. Kopeliovich and A.V. Tarasov, Phys. Rev. D62 (2000) 094022.

[29] L. Frankfurt, L. Gerland, M. Strikman and M.Zhalov, Phys. Rev. C68 (2003) 044602;

L. Gerland, L. Frankfurt, M. Strikman and H. Stocker, Phys. Rev. C69 (2004) 014904.

[30] Review of Particle Properties, K. Hagiwara et al., Phys. Rev. D66 (2002) 010001.

[31] F.E. Close and G.A. Schuler, Phys. Lett. B464 (1999) 279.

[32] A.D. Martin, M.G. Ryskin and V.A. Khoze, Phys. Rev. D56 (1997) 5867.

[33] V.A. Khoze, A.D. Martin and M.G. Ryskin, hep-ph/0006005 in Proc. of 8th Int. Workshop on Deep Inelastic Scattering and QCD (DIS2000), Liverpool, eds. J. Gracey and T. Greenshaw (World Scientific, 2001), p.592.

[34] V.A. Khoze, A.D. Martin and M.G. Ryskin, Nucl. Phys. Proc. Suppl. 99B (2001) 188.

[35] L.D. Landau, Dokl. Akad. Nauk SSSR 60 (1948) 213;

C.N. Yang, Phys. Rev. 77 (1950) 242.

[36] A.D. Martin, R.G. Roberts, W.J. Stirling and R.S. Thorne, Eur. Phys. J. C14 (2000) 133.

[37] E.J. Eichten and C. Quigg, Phys. Rev. D52 (1995) 1726.

[38] R. Barbieri, M. Caffo, R. Gatto and E. Remiddi, Phys. Lett. B95 (1980) 93; Nucl. Phys. B192 (1981) 61.

[39] S. Kim, Nucl. Phys. Proc. Suppl. 47 (1996) 437.

[40] G.T. Bodwin, D.K. Sinclair and S. Kim, Nucl. Phys. Proc. Suppl. 34 (1994) 434; 42 (1995) 306.

[41] G.T. Bodwin, D.K. Sinclair and S. Kim, Phys. Rev. D65 (2002) 054504.

[42] Belle Collaboration: C.-K. Choi et al., Phys. Rev. Lett. 91 (2003) 262001, arXiv:hep-ex/0309032 CDF Collaboration: D. Acosta et al., arXiv:hep-ex/0312021, submitted to Phys. Rev. Lett.

[43] F.E. Close and P.R. Page, Phys. Lett. B578 (2004) 119. 